\title{
Sevenfold Coordinated MgSe: Experimental Internal Atom Position Determination to 146 GPa, Diffraction Studies to 202 GPa, and Theoretical Studies to 500 GPa
}

\author{
Arthur L. Ruoff, Ting Li, Allen C. Ho, Min-Fan Pai, Huan Luo, Ray G. Greene, and Chandrabhas Narayana \\ Department of Materials Science And Engineering, Cornell University, Ithaca, New York 14853 \\ Jay C. Molstad, Steven S. Trail, and Francis J. DiSalvo, Jr. \\ Department of Chemistry, Cornell University, Ithaca, New York 14853 \\ P.E. van Camp \\ Department of Physics, University of Antwerpen Groenenborggklaan, 171, B-2000, Antwerpen, Belgium
}

(Received 14 October 1997)

\begin{abstract}
$\mathrm{MgSe}$ has been studied using energy dispersive x-ray diffraction to $202 \mathrm{GPa}$ and local density approximation and ultrasoft pseudopotentials to $500 \mathrm{GPa}$. MgSe undergoes a "continuous" phase transformation from the rocksalt to FeSi (B28) beginning at around $99 \pm 8 \mathrm{GPa}$ and approaching sevenfold coordination at $202 \mathrm{GPa}$. Theoretical computation finds the $B 28$ transition beginning at $58 \mathrm{GPa}$ followed by a transition to an orthorhombic distortion of the $B 2$ structure at $429 \mathrm{GPa}$. [S0031-9007(98)07261-5]
\end{abstract}

PACS numbers: $64.70 . \mathrm{Kb}, 61.10 .-\mathrm{i}, 62.50 .+\mathrm{p}, 64.30 .+\mathrm{t}$

The chalcogenides of $\mathrm{Ba}, \mathrm{Sr}$, and $\mathrm{Ca}$ have the sixfold coordinated $\mathrm{NaCl}$ structure $(B 1)$ and transform at high pressure to the eightfold coordinated $\mathrm{CsCl}$ structure $(B 2)$ except for $\mathrm{BaO}$ which transforms to a distorted $\mathrm{CsCl}$ structure [1]. The compounds of $\mathrm{MgSe}, \mathrm{MgS}$, and $\mathrm{MgO}$ also have the $B 1$ structure. $\mathrm{MgO}$ is of great interest to geophysicists as it is one likely component of the lower mantle [2]. It has been calculated that there will be a $B 1 \rightarrow$ cesium chloride (B2) structure transformation at around $600 \mathrm{GPa}$ for $\mathrm{MgO}[3,4]$ and at $231 \mathrm{GPa}$ for $\mathrm{MgS}$ [5]. From an empirical map based on the ratio of the cation radius to the anion radius versus the logarithm of the pressure of the chalcogenides of $\mathrm{Ba}, \mathrm{Sr}$, and $\mathrm{Ca}$, the $B 1$ to $B 2$ transformation is expected as follows: $\mathrm{MgSe}$ (175 GPa), MgS (172 GPa), MgO (210 GPa), if no other transition intervenes [1].

The MgSe powder sample was prepared from the reaction of $\mathrm{Mg}$ shavings (Strem Chemicals 99.9\%) and selenium pieces (Aldrich $99.9 \%$ ), placed in a boron nitride crucible, then evacuated and sealed in a quartz tube and heated at $1023 \mathrm{~K}$ for 24 hours. The x-ray powder diffraction of the sample showed it to be single phase, with a cubic cell with $a=5.466 \AA$; the literature value is $a=5.462 \AA$. The samples were handled in a dry argon environment to avoid exposure to air. The first experiment used gold [6] as a pressure marker and reached $60 \mathrm{GPa}$. The second experiment used platinum [7] as a pressure marker and reached $146 \mathrm{GPa}$. Data were collected on downloading to $109 \mathrm{GPa}$ with no hysteresis. The equation of state (EOS) was determined from these two experiments. In the third experiment which reached $202 \mathrm{GPa}$ the pressure was determined from the EOS of $\mathrm{MgSe}$ so that pressure marker peaks did not obfuscate the x-ray pattern. In the last experiment, the diamond anvil cell (DAC) was rotated about the x-ray beam, clockwise and anticlockwise by $360^{\circ}$, alternately, while the experiment was in progress, in an effort to reduce the effect of texturing in the sample. No pressure medium was used in any of these experiments.

Energy dispersive $\mathrm{x}$-ray diffraction (EDXD) experiments were performed at the Cornell High Energy Synchrotron Source (CHESS) to get the x-ray diffraction spectra and EOS of the MgSe sample [8]. A set of tungsten apertures collimated the incident $\mathrm{X}$-ray beam to $20 \times$ $20 \mu \mathrm{m}^{2}$ in the first two experiments, and $17 \times 17 \mu \mathrm{m}^{2}$ in the third experiment. The diffraction geometry was calibrated with gold foil. The typical collection times for a spectrum were approximately 20 minutes in the first experiment, 60 minutes in the second experiment, and around 1 to 2 hours in the third experiment. On loading from 0 to $96 \mathrm{GPa}$ all spectra indexed well to the initial $B 1$ phase. Figure 1(a) shows a typical EDXD spectrum of MgSe near $35 \mathrm{GPa}$ indexed to the $B 1$ structure [9]. For pressures greater than $96 \mathrm{GPa}$, new sample diffraction lines appear in addition to those of the rocksalt pattern, indicating a phase transition. Figure 1(b) shows an EDXD spectrum of $\mathrm{MgSe}$ at $99 \mathrm{GPa}$ with arrows pointing out the extra peaks. The intensities of the new peaks increased gradually with pressure. It was found that a simple cubic lattice with the same lattice constant as that of the rocksalt phase produces all the interplanar spacings of the observed peaks. An excellent fit (of the 32 data points to $146 \mathrm{GPa}$ ) exists with the two-parameter EOS of Birch [10]

$$
\begin{aligned}
P= & (3 / 2) B_{0}\left(x^{7 / 3}-x^{5 / 3}\right) \\
& \times\left[1+(3 / 4)\left(B_{0}^{\prime}-4\right)\left(x^{2 / 3}-1\right)\right],
\end{aligned}
$$

where $x=V_{0} / V, B_{0}$ is the isothermal bulk modulus at zero pressure, and $B_{0}^{\prime}$ is the pressure derivative of the isothermal bulk modulus evaluated at zero pressure. The resulting values of the two parameters, applicable to 


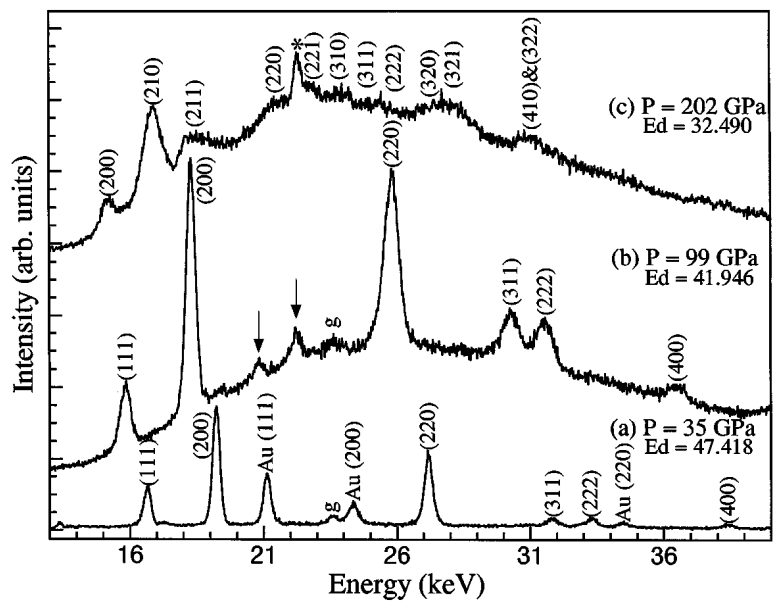

FIG. 1. EDXD diffraction spectra of MgSe. $g$ is gasket; $\mathrm{Au}$ is gold; all other labeled peaks are the sample. $E d$ is in the unit of keV $\AA$. (a) Original $B 1$ structure near $35 \mathrm{GPa}$. (b) $99 \mathrm{GPa}$, new peaks shown with arrows. (c) $202 \mathrm{GPa}$, showing $B 28$ phase. In $(b)$ and $(c)$ pressure was determined from the measured lattice parameter of $\mathrm{MgSe}$ and its EOS, and also the sample was rotated about the x-ray beam (see text). The $*$ in curve (c) may represent the disallowed diamond peak (112) since it would come from the highly strained tip region of the diamond. We note that this peak and four other peaks from diamond at energies above $45 \mathrm{keV}$ are all very much sharper than the peaks from $\mathrm{MgSe}$. These five peaks give $a=3.573 \pm 0.002 \AA$. We do not rule out the possibility that the $*$ peak in curve $(c)$ is a new peak of a second phase.

both phases, are $B_{0}=62.8 \pm 1.6 \mathrm{GPa}$ and $B_{0}^{\prime}=4.1 \pm$ 0.1 with $V_{0}(B 28)=V_{0}(B 1)$. The $B 28$ structure $[11,12]$ was assigned to the high pressure phase; the magnesium atoms are located at $u u u ; u+1 / 2,1 / 2-u, \bar{u} ; \bar{u}, u+$ $1 / 2,1 / 2-u ; 1 / 2-u, \bar{u}, u+1 / 2$ and selenium atoms at $w w w ; w+1 / 2,1 / 2-w, \bar{w} ; \bar{w}, w+1 / 2,1 / 2-w$; $1 / 2-w, \bar{w}, w+1 / 2$ [12]. If $u=0$ and $w=1 / 2$, this is the $\mathrm{NaCl}(B 1)$ structure. The experimental values of $u$ and $w$ are obtained by minimization of the Bragg $R_{B}$. See Table I. Here $R_{B}=\left(\sum I_{\mathrm{obs}}-I_{\text {calc }}\right) / \sum I_{\mathrm{obs}}$ (see Table 1.3 of Ref. [13]). At $146 \mathrm{GPa}$, there is a sharp minimum at $u=0.0481, w=0.4520$.

As texturing was evident in the first two experiments, a third experiment was performed in which the pressure vessel and hence the sample were rotated about the x-ray beam to average (partially) the effect of texturing. This is similar to the use of image plates in angle dispersive
TABLE I. List of the observed interplanar spacings $(d)$ and relative intensities $(I)^{*}$ of $\mathrm{MgSe}$ at $146 \mathrm{GPa}$ along with the theoretical corresponding values based on the FeSi structure. The fitted lattice parameter is $4.450 \pm 0.002$. The energyinterplanar spacing product was $E d=42.590 \mathrm{keV} \AA$.

\begin{tabular}{ccccc}
\hline \hline$(h k l)$ & $d_{\text {obs }}(\AA)$ & $d_{\text {calc }}(\AA)$ & $I_{\text {obs }}(\%)$ & $I_{\text {calc }}(\%)$ \\
\hline$(110)$ & 3.143 & 3.147 & 3 & 9 \\
$(111)$ & 2.576 & 2.569 & 38 & 49 \\
$(200)$ & 2.224 & 2.225 & 100 & 100 \\
$(210)$ & 1.991 & 1.990 & 64 & 64 \\
$(211)$ & 1.813 & 1.817 & 12 & 12 \\
$(220)$ & 1.578 & 1.573 & 28 & 38 \\
$(221)$ & 1.485 & 1.483 & 20 & 25 \\
$(310)$ & 1.408 & 1.407 & 9 & 7 \\
$(311)$ & 1.342 & 1.342 & 7 & 6 \\
$(222)$ & 1.288 & 1.285 & 18 & 6 \\
$(321)$ & 1.190 & 1.189 & 14 & 10 \\
$(410)+(322)$ & 1.081 & 1.079 & 3 & 9 \\
$(421)$ & 0.974 & 0.971 & 2 & 5 \\
\hline \hline
\end{tabular}

${ }^{*}$ The $(h 00)$ peaks, for odd values of $h$, of $B 28$ always have $I=0$. $R_{B}$ is based on all the peaks from (110) to (521) including those of zero observed intensities. The minimum is at $u=0.048 \pm$ 0.003 and $w=0.452 \pm 0.003$ with $R_{B}=0.24$. In other patterns at different pressures the following peaks were also present: (320), (400), (331), (420), (332), (422), (510), (511), (423), and (521).

$\mathrm{X}$-ray diffraction (ADXD). It plays the same role as does averaging over $360^{\circ}$ in a Debye Scherrer ring on an image plate in ADXD [14]. The diamond tips used had (100) faces. When the cell is rotated single crystal peaks from the diamond can occur. At the angles used no allowed diamond peaks existed below $45 \mathrm{keV}$. However, allowed peaks were seen above $45 \mathrm{keV}$. Figure 1(c) shows a diffraction pattern at $202 \mathrm{GPa}$. At $170 \mathrm{GPa}$ and above, the (210) peak is the strongest peak. Figure 2 shows experimental data at $146 \mathrm{GPa}$ and the calculated peaks. The background intensity at a specific pressure as a function of energy $E$ was fitted to the expression

$$
I_{B}(E)=\alpha \exp \left[-\left(\beta / E^{3}-\gamma / E^{4}\right)\right] \exp (-\delta E),
$$

and this was subtracted from the total intensity to give the corrected pattern. The peaks were fitted with Gaussians, to obtain peak positions (and hence the interplanar spacing) and the FWHM's. The theoretical relative intensity pattern for a case where the 200 peak is strongest was calculated for the sum of all the theoretical peaks

$$
\left(I_{h k l} / I_{200}\right)=\left[I_{B}(h k l) M_{h k l} F_{h k l}^{2} I_{200} E_{200}^{2}\right] /\left[I_{B}(200) M_{200} F_{200}^{2} I_{h k l} E_{h k l}^{2}\right],
$$

where $M$ is multiplicity, $F$ is the cell scattering factor, and $I$ is due to the incoherent Compton scattering from the diamond.

At $202 \mathrm{GPa}$ there is a sharp minimum at $u=0.0828$ and $w=0.4173$, which we round off to $0.08 \pm 0.01$ and $0.42 \pm 0.01$. This corresponds to six atoms at $0.53 a \pm$ $0.01 a$ and one at $0.58 a \pm 0.01 a$. The theoretical values at $202 \mathrm{GPa}$ are $u=0.0759$ and $w=0.4136$ (see
Fig. 3). For seven equidistant neighbors $u=0.095495$ and $w=0.404508$, the bond distance is $0.5352 a$, and $w=1 / 2-u$.

The calculations were performed in the framework of the local density approximation using ultrasoft pseudopotentials [15] and a large plane wave basis. For the exchange-correlation part the authors used the CeperleyAlder expression [16] as parametrized by Perdew and 


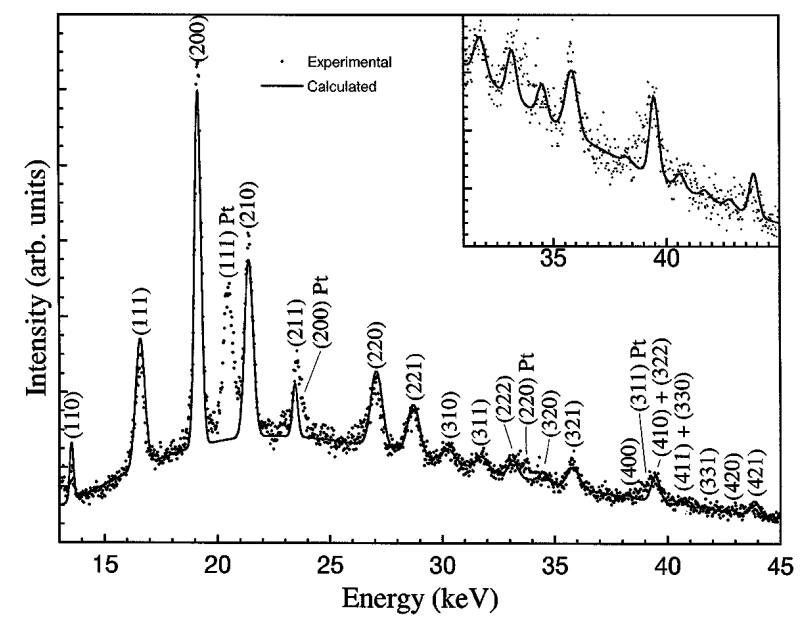

FIG. 2. Experimental and theoretical x-ray patterns (based on minimization of $R_{B}$ with respect to $u$ and $w$ ) for the $B 28$ structure of $\mathrm{MgSe}$ at $146 \mathrm{GPa}$. The inset shows amplified intensities from 31 to $45 \mathrm{keV}$ and the two energy axes coincide. A peak position in the inset is at the same energy as in the main figure.

Zunger [17]. The optimization of the internal parameters and of the lattice parameters was performed with the Broyden-Fletcher-Goldfarb-Shanno algorithm [17]. The calculated total energies were then fitted to the twoparameter Birch EOS from which the bulk modulus and its pressure derivative were determined. The main difference from previous work [18] is that now the core radii were taken a lot smaller so that calculations can be performed to higher pressures. Moreover, a larger cutoff of $64 \mathrm{Ry}$ was used and more structures were considered, e.g., rocksalt $(B 1)$, cesium chloride $(B 2)$, zinc blende $(B 3)$, wurtzite $(B 4)$, nickel arsenide $(B 8)$, iron silicide (B28), and simple tetragonal (ST2) and orthorhombic (SO2) structures [19]. SO2 is a distortion of ST2, which in turn is a distortion of $B 2$. Table II shows the lat-

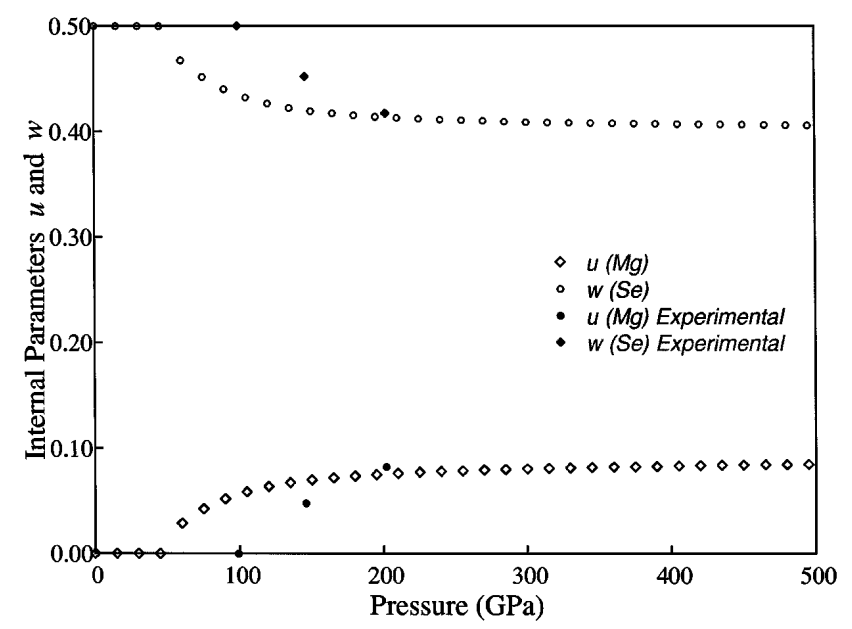

FIG. 3. Calculated internal atomic positions of $u$ of $\mathrm{Mg}$ and $w$ of $\mathrm{Se}$ as a function of pressure and experimental values at 99, 146, and $202 \mathrm{GPa}$. The data at $202 \mathrm{GPa}$ have a larger error bar than the other two points.
TABLE II. Calculated lattice constant $a$, bulk modulus $B_{0}$, and its pressure derivative $B_{0}^{\prime}$ for five phases of $\mathrm{MgSe}$, namely, rocksalt $(B 1)$, cesium chloride $(B 2)$, iron silicide $(B 28)$, simple tetragonal (ST2), and simple orthorhombic (SO2).

\begin{tabular}{cccccc}
\hline \hline & $B 1$ & $B 2$ & $B 28$ & $\mathrm{ST} 2$ & $\mathrm{SO} 2$ \\
\hline$a$ & 5.4975 & 3.4413 & 5.4967 & $3.4133^{\mathrm{a}}$ & $3.4133^{\mathrm{a}}$ \\
$B_{0}$ & 68.12 & 63.68 & 51.11 & 60.79 & 61.55 \\
$B_{0}^{\prime}$ & 4.08 & 4.04 & 4.10 & 4.08 & 4.07 \\
\hline \hline
\end{tabular}

${ }^{\text {a }}$ These structures tranform to $B 2$ at $P=0$.

tice constant, bulk modulus, and its pressure derivative for five structures. Table III shows the calculated transition pressures, the volume change at the transitions, and the energy difference between the two structures at the transitions. For the $\mathrm{SO} 2$ structure at the transition pressure (429.3 GPa) we find a $b / a$ value slightly smaller than 1 and a $c / a$ value slightly larger than 1 . Thus $\mathrm{SO} 2$ is a slightly distorted $\mathrm{CsCl}$ structure. It is always more stable than $B 2$, at high pressure. It is possible that thermal effects not considered here would make the more symmetrical $\mathrm{CsCl}$ structure the stable high pressure phase at room temperature. Were the ST2 and SO2 structure absent, a $B 28$ to $B 2$ equilibrium transition point would exist at $472 \mathrm{GPa}$ while if $B 28$ were also absent the $B 1$ to $B 2$ transition would be at $170 \mathrm{GPa}$.

Finally, a comparison of the experimental and theoretical results is made. Using the Birch EOS, the experimental values of $B_{0}=62.8 \mathrm{GPa}$ and $B_{0}^{\prime}=4.1$ agree fairly well with the calculated values of $68.12 \mathrm{GPa}$ and 4.08 , respectively. Experimentally it was found, at a pressure of $202 \mathrm{GPa}$, that the internal atomic positions $u=0.08$ and $w=0.42$ are near the theoretically calculated values of 0.0759 and 0.4136 , respectively. At $202 \mathrm{GPa}, \mathrm{MgSe}$ is close to the sevenfold structure as in AuBe.

The present experimental determination was limited by broad peak widths owing to strain broadening and pressure gradients (caused by the large yield stress; for comparison $\mathrm{MgO}$ has a yield strength of at least $11 \mathrm{GPa}$ at $227 \mathrm{GPa}[20])$. Thus, in the earliest stages of the transformation because of the presence of a pressure gradient and a finite beam size, the diffraction pattern would be partially from the $B 1$ phase and partially from the $B 28$ phase. This would be followed by a stage in which only $B 28$ was present but with a large variation of $u$ and $w$ across the finite beam since these vary rapidly near the transition pressure. In future experiments, to determine $u$ and $w$ in the range in which their variation with pressure

TABLE III. Calculated transition pressure $P$ (in GPa), the volume change $\Delta V$ (in $\%$ ), and the energy difference $\Delta E$ (in $\mathrm{meV}$ ) for the phase transition from $B 1$ to $B 28$ and $B 28$ to SO2.

\begin{tabular}{ccc}
\hline \hline & $B 1$ to $B 28$ & $B 28$ to $\mathrm{SO} 2$ \\
\hline$P$ & 57.0 & 429.3 \\
$\Delta V$ & 1.5 & 1.2 \\
$\Delta E$ & 74.0 & 245.9 \\
\hline \hline
\end{tabular}


is large, a hydrostatic medium, such as helium, should be used to minimize these effects. We intend to carry out such studies. If broadening occurring during the transformation is small, then the intrinsic detector peak width of $0.145 \sqrt{E(\mathrm{keV}) / 5}$ would apply. Should this prove to be a limitation, then the detector needs to be replaced with one of much higher resolution such as the microcalorimeter (bolometer) detectors which are undergoing rapid development [21]. With near hydrostatic pressure, and sensitive detectors, and possibly with sample rotation, EDXD has a bright future as a tool for structural determination including accurate determination of internal atom positions.

Within our experimental error limits there is no noticeable volume change in the $B 1$ to $B 28$ transition, so it is likely that the transformation is second order (although theoretical calculations show a small volume change). Based on symmetry arguments, the $X_{5}^{-}$irreducible representation can drive such a transition from $F m \overline{3} m$ (225) to $T^{4} P 2{ }_{1} 3$ (198) [22]. Another fact that gives some reinforcement to this conclusion is that the reverse transformation during unloading proceeded without any observable hysteresis.

The distorted rocksalt structure discovered in $\mathrm{MgSe}$ at around one megabar is the first example of a sixfold coordinated rocksalt structure transforming to a sevenfold coordinated iron silicide structure at high pressure. It is interesting to note that $\mathrm{FeSi}$ is a narrow gap semiconductor at low temperature [23] and becomes metallic over $300 \mathrm{~K}$ [24], which is consistent with the expected metallization of $\mathrm{MgSe}$ at high pressure. It would be instructive to consider $B 28$ as a possible structure in theoretical calculations for $\mathrm{MgS}$ and $\mathrm{MgO}$ as well. The potential significance of the $B 28$ structure lies in the conjecture that if the $B 28$ structure has a substantially lower transition pressure when compared to $B 2$ for $\mathrm{MgO}$, it will have important geological implications [20].

We thank the CHESS staff for their technical assistance and the National Science Foundation for support of this research through Grant No. DMR 9530634.

[1] C. Narayana, V. J. Nesamony, and A. L. Ruoff, Phys. Rev. B 56, 14338 (1997).
[2] F. Birch, J. Geophys. Res. 57, 227 (1952).

[3] K. J. Chang and M.L. Cohen, Phys. Rev. B 30, 4774 (1984).

[4] M.D. Jackson and R. G. Gordon, Phys. Rev. B 38, 5654 (1988).

[5] P.E. van Camp, V.E. van Doren, and J. L. Martins, Phys. Status Solidi B 190, 193 (1995).

[6] J. C. Jamieson, J. Fritz, and M. H. Manghnani, Adv. Earth Planet. Sci. 12, 27 (1980).

[7] N. C. Holmes, J. A. Moriarty, G. R. Gathers, and W. J. Nellis, J. Appl. Phys. 66, 2962 (1989).

[8] M. Baublitz, Jr., V. Arnold, and A. L. Ruoff, Rev. Sci. Instrum. 52, 1616 (1981).

[9] C. Narayana, A. C. Ho, M-F. Pai, and A. L. Ruoff, in Review of High Pressure Science and Technology, edited by M. Nakahara (to be published).

[10] F. Birch, J. Geophys. Res. 83, 1257 (1978).

[11] L. Pauling and A. M. Soldate, Acta. Crystallogr. 1, 212 (1948).

[12] B. D. Cullity, Trans. AIME 171, 396 (1947).

[13] R.A. Young, The Rietveld Method (Oxford University Press, New York, 1993), p. 22.

[14] Y. Fujii, K. Hose, Y. Ohishi, N. Hamaya, K. Takemura, O. Shimomura, T. Kigegawa, Y. Amemiya, and T. Matushita, Phys. Rev. Lett. 63, 536 (1989).

[15] N. Troullier and J.L. Martins, Solid State Commun. 74, 613 (1990); Phys. Rev. B 43, 1993 (1991).

[16] D. Ceperley and B. Alder, Phys. Rev. Lett. 45, 566 (1980).

[17] J. P. Perdew and A. Zunger, Phys. Rev. B 23, 5048 (1981).

[18] J. Olsen and P. Jorgensen, J. Chem. Phys. 77, 6109 (1982).

[19] P.E. van Camp, V.E. van Doren, and J. L. Martins, Phys. Rev. B 55, 775 (1997).

[20] T. S. Duffy, R. J. Hemley, and H-K. Mao, Phys. Rev. Lett. 74, 1371 (1995).

[21] D. A. Wollman, G. C. Hilton, K. D. Irwin, and J. Martins, in Proceedings of Microscopy and Microanalysis, edited by G. N. Bailey, J. M. Corbett, R. V.W. Dimlich, J.R. Michael, and N.J. Zaluzec (San Francisco Press, San Francisco, 1996), p. 488.

[22] H. T. Stokes and D. M. Hatch, Isotropy Subgroups of the 230 Crystallographic Space Groups (World Scientific, Teanech, NJ, 1988).

[23] V. Jaccarino, G. K. Wertheim, J.H. Wernick, L. R. Walker, and S. Arajs, Phys. Rev. 160, 476 (1967).

[24] R. Wolfe, J. H. Wernick, and S. E. Haszko, Phys. Lett. 19, 449 (1965). 